\title{
Electrochemical Promotion in Emission Control Catalysis
}

\author{
R. M. Lambert, I. R. Harkness, I. V. Yentekakis* and C. G. Vayenas* \\ Chemistry Department, Cambridge University, Cambridge CB2 1EW, England, \\ *ICE/HT University of Patras, GR-26500 Greece
}

\begin{abstract}
Electrochemical promotion for the catalytic reduction of NO by $\mathrm{CO}$ and of NO by ethylene over a Pt catalyst are reported for the first time. Both reactions are of importance in the catalytic control of automotive emissions and both exhibit strong rate enhancement when $\mathrm{Na}$ is pumped to the Pt catalyst electrode from a $\beta$ " solid electrolyte. Complementary data obtained with a $\mathrm{Pt}(111) / \mathrm{Na}$ model system indicate that electrochemically-pumped $\mathrm{Na}$ acts by inducing dissociation of chemisorbed NO, which is the reaction initiating step.
\end{abstract}

\section{Introduction}

Electrochemical pumping of a metal catalyst film in contact with a solid electrolyte can induce very large changes in catalytic rate and selectivity. The processes involved are strongly non-Faradaic and the phenomena at work are not fully understood; it has been proposed that the principal effect involves changes in chemisorptive properties caused by workfunction changes induced by spillover of pumped ions from electrolyte to catalyst. The first studies with $\beta$ " alumina as the electrolyte were carried out by Vayenas et al on the Pt-catalysed oxidation of ethylene [1]. We have quantitatively reproduced their results, and, in addition, some new features were observed. We have also investigated two reactions which have never been studied before with respect to electrochemical promotion (EP): the reaction of $\mathrm{CO}$ with NO, and of NO with ethylene. Both are directly relevant to emission control catalysis and both display strong electrochemical promotion. The first exhibits instabilities, bifurcation, hysteresis and rate oscillations; the second shows a massively enhanced rate under EP conditions Atmospheric pressure EP studies were carried out in a CSTR electrochemical reactor with product analysis by means of a quadrupole mass spectrometer. Most observations were carried out in potentiostatic mode by following the effect of $\mathrm{V}_{w r}$ on the reaction rate.

Complemenatry kinetic and spectroscopic measurements were carried out on $\mathrm{Pt}(111)$ single crystal model catalyst which could be translated between a UHV cham- ber (XPS, TPD, Na dosing, Ar+ etching) and a pressure cell $\left(30 \mathrm{~cm}^{3}\right)$ which was operated as a differential batch reactor. Na dosing was by means of a collimated evaporation source, Na coverage calibration being achieved by TPD and XPS. In the kinetic experiments, partial pressures, temperature regime and $\mathrm{Na}$ coverages were chosen so as to reproduce as closely as possible the conditions in the electrochemical CSTR system.

1.1 Ethylene oxidation. The observed open circuit turnover frequency of $\approx 1$ at $280^{\circ} \mathrm{C}$ compares well with values obtained under similar conditions in earlier work by Vayenas et al [1]. However, some interesting new features were also observed. In particular, the rise in rate at moderate sodium coverages before the precipitous fall at more negative biases was not previously seen. We ascribe this rise to Na-induced strengthening of the Pt-O bond which leads to an increase in oxygen coverage (and hence in reaction rate) in a regime where the reaction exhibits positive order in oxygen. The sharp fall-off at high Na coverages $\left(\mathrm{V}_{\mathrm{Wr}} \ll 0\right)$ is thought to be due to two effects which work in the same direction: (i) $\mathrm{Na}$ lowers the surface work function causing an increase in the strength of the Pt-O bond thereby increasing the activation energy of the rate limiting step [5]. (ii) the accumulation of surface compounds of $\mathrm{Na}$ leads to extensive blocking of previously active surface sites. This latter proposal is supported by the single crystal data (see below).

TPD analysis of the $\mathrm{Pt}(111)$ model catalysts after 
running the reaction over an initially clean surface (3.6 $\mathrm{kPa} \mathrm{O}_{2}, 1.3 \mathrm{kPa} \mathrm{C}_{2} \mathrm{H}_{4}$ ) showed that the only species retained on the surface after this procedure was carbon monoxide: no desorption of water or either reactant was detectable. It is important to note that under the reaction conditions employed here, there was no detectable production of $\mathrm{CO}$ during catalytic turnover in the reactor cell: the only products found were $\mathrm{CO}_{2}$ and $\mathrm{H}_{2} \mathrm{O}$. Control experiments using ${ }^{13} \mathrm{CO}$ clearly demonstrated that post-reaction TPD analysis does indeed convey relevant information about the surface condition at the end of the reaction.

Kinetic parameters extracted from the experiments with the model catalyst yield activation energy values which lie in the range expected if the $\mathrm{CO}+\mathrm{O}$ elementary reaction step is rate limiting [6]. This agrees with the observation of an appreciable CO coverage on the surface after reaction. Encouragingly, he trends in kinetic parameters for $\mathrm{Pt}(111) / \mathrm{Na}$ follow those observed in the electrochemical CSTR system. Post-reaction TPD spectra acquired after ethylene oxidation on the $\operatorname{Pt}(111)$ surface after first dosing the latter with varying coverages of $\mathrm{Na}$ exhibit major differences compared to the $\mathrm{Na}$-free case. Simultaneous peaks at $23 \mathrm{amu}\left(\mathrm{Na}^{+}\right)$and $44 \mathrm{amu}$ $\left(\mathrm{CO}_{2}^{+}\right)$are observed: we ascribe these to the presence of an alkali surface carbonate - a conclusion which is supported by the XAE and XP spectra. Thus the X-ray excited Na KLL Auger electron spectra obtained before and after the reaction indicate a loss of sodium superficial area and a change in the chemical nature of the sodium. Note that these results provide an explanation for the suppression at high temperatures of the strong poisoning normally observed in the CSTR EP experiments at $V_{w r}$ $\ll 0$ : the Na surface compounds are volatilised.

$1.2 \mathrm{CO}+\mathrm{NO}$ reaction. The reaction of $\mathrm{CO}$ with $\mathrm{NO}$ over Pt surfaces has been studied intensively because of its importance in emission control catalysis and because it exhibits fundamentally interesting properties, including rate oscillations. The reaction mechanism is controversial: some authors claim that Pt-catalysed NO dissociation is the rate limiting step, while others claim that this is inconsistent with observed kinetic behaviour invoking the bimolecular $\mathrm{NO}+\mathrm{CO}->\mathrm{N}+\mathrm{CO}_{2}$ reaction as the rate limiting step. In the majority of studies, at low pressure, the former is accepted, however, at high pressure the nature of the ratelimiting step has still to be resolved. Ours is the first investigation of the reaction under conditions of electrochemical promotion.
Depending on the conditions, the system exhibits bifurcation instabilities, hysteresis (as a function of $\mathrm{V}_{\mathbf{w r}}$ ) and rate oscillations. The general form of the rate behaviour is that there are 2 branches with rates that differ by a factor of $\approx 4$. The lower rate branch which is the stable state on the sodium free surface (high positive $\mathrm{V}_{\mathrm{wr}}$ ) is promoted by increasing sodium coverage, the higher rate branch inhibited by sodium. There is some evidence that the low rate branch is also inhibited at very negative $V_{w r}$ values, but generally it is unstable at those potentials. Switching between the two states is dependent on the gas composition, with the lower rate state being less stable the higher the partial pressure of CO. Significant hysteresis is observed with changing between the rate branches being very dependent on whether $\mathrm{V}_{\mathbf{w r}}$ is increasing or decreasing.

As the system switched from low rate to high rate behaviour, a negative current flowed in the w-r circuit, i.e., $\mathrm{Na}^{+}$was pumped towards the $\mathrm{Pt}$ catalyst electrode. This current flow occurs because the sample is biased in the potentiostatic mode, where the sample potential, which is equivalent to its work function, is held constant. The fact that the potentiostat has to supply $\mathrm{Na}$ to the catalyst when the rate changes from low to high rate implies that the coverage of an electron-withdrawing adsorbate has increased. In this reaction system the likely electron-withdrawing species are $\mathrm{O}$ or $\mathrm{N}$ rather than NO or CO. This suggests that the EP effect is associated with Na-induced dissociation of NO. That is, Na triggers the reaction by markedly decreasing the activation energy for the otherwise rate-limiting dissociation of adsorbed NO.

Results obtained with the $\mathrm{Pt}(111)$ model catalysts show that when $\mathrm{Na}$ was present on the surface, $\mathrm{CO}_{2}$ evolution was always observed in the desorption spectra, consistent with increased dissociation of NO in the reactor and a higher coverage of $\mathrm{O}_{\mathrm{a}}$ prior to the TPD sweep. Furthermore, the 23 amu spectra show clear evidence for the formation of stable surface carbonates which could account for the poisoning behaviour which is observed under electrochemical conditions at $V_{w r} \ll 0$ (high Na levels). The TPD data also indicate that even with initial $\mathrm{Na}$ doses of the order of a monolayer, under reaction conditions a substantial fraction of the surface consists of bare Pt sites due to at least partial agglomeration of Na-containing surface compounds. This is entirely consistent with the XPS data which point to the same conclusion - namely that agglomeration of the $\mathrm{Na}$ 
surface compounds occurs under reaction conditions. This enables us to understand why (in comparison to ethylene oxidation) the CO+NO reaction is not very strongly poisoned at $\mathrm{V}_{\mathrm{wr}} \ll 0$. The $\mathrm{Na}$ surface complexes produced in the former case are stabilised by interaction with the Pt surface which they therefore cover as a monolayer; in the present case, strongly ionic Na oxides may be produced and the associated 3-D Madelung energy favours crystallite agglomeration rather than 2-D wetting.

\section{References}

[1] C.G. Vayenas, S. Bebelis, and S. Ladas, Nature, 343 No. $6259625-625$ (1990) C. G. Vayenas, S. Bebelis, I. V Yentekakis and H.-G. Lintz Catalysis Today 11 303-442 (1992)

[2] C.G. Vayenas, S. Bebelis and M. Despotopoulou,
J. Catal. 128, 415-435 (1991)

[3] S. Bebelis and C.G. Vayenas J. Catal.., 118, 125146 (1989)

[4] J. Hölzl and F.K. Schulte, "Work Function of Metals" in Solid Surface Physics, Springer-Verlag, Berlin, pp 1-150 (1979)

[5] W. Schroder and J. Hölzl, Solid State Commun., 24, 777 (1977)

[6] S.Bebelis and C.G. Vayenas, J. Catal., 138, 570 (1992)

[7] C.T. Campbell, G. Ertl, H. Kuipers and J. Segner, J. Chem. Phys., 73, 5862 (1980).

Paper presented at the 1st Euroconference on Solid State Ionics, Zakynthos, Greece, 11 - 18 Sept. 1994.

Manuscript received 11 Sept. 1994 